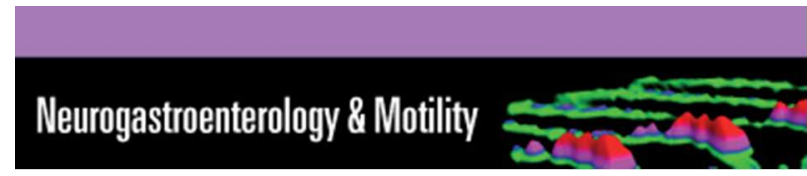

\title{
Localization and expression of TRPV1 and TRPA1 in the human oropharynx and larynx
}

\begin{tabular}{|c|c|}
\hline Journal: & Neurogastroenterology and Motility \\
\hline Manuscript ID: & NMO-00206-2015.R1 \\
\hline Manuscript Type: & Original Article \\
\hline Date Submitted by the Author: & $\mathrm{n} / \mathrm{a}$ \\
\hline Complete List of Authors: & $\begin{array}{l}\text { Alvarez-Berdugo, Daniel; Hospital de Mataró, Gastrointestinal Physiology } \\
\text { Laboratory, Department of Surgery } \\
\text { Rofes, Laia; Centro de Investigación Biomédica en Red de Enfermedades } \\
\text { Hepáticas y Digestivas, Instituto de Salud Carlos III, } \\
\text { Farre, Ricard; Centro de Investigación Biomédica en Red de Enfermedades } \\
\text { Hepáticas y Digestivas, Instituto de Salud Carlos III, ; KU Leuven - } \\
\text { University of Leuven, Translational Research Center for Gastrointestinal } \\
\text { Disorders } \\
\text { Casamitjana, Josep Francesc; Hospital de Mataró, Consorci Sanitari del } \\
\text { Maresme, ENT department } \\
\text { Enrique, Ana; Hospital de Mataró, Consorci Sanitari del Maresme, ENT } \\
\text { department } \\
\text { Chamizo, Juan; Hospital de Mataró, Consorci Sanitari del Maresme, ENT } \\
\text { department } \\
\text { Padrón, Andreina; Hospital de Mataró, Consorci Sanitari del Maresme, } \\
\text { Department of Pathology } \\
\text { Navarro, Xavier; Universitat Autònoma de Barcelona, and Centro de } \\
\text { Investigación Biomédica en Red sobre Enfermedades Neurodegenerativas, } \\
\text { Department of Cell Biology, Physiology and Immunology, Institute of } \\
\text { Neurosciences } \\
\text { Clavé, Pere; Hospital de Mataró, Gastrointestinal Physiology Laboratory,' } \\
\text { Department of Surgery; Fundació Institut de Investigació Germans Trias i } \\
\text { Pujol, ; Centro de Investigación Biomédica en Red de Enfermedades } \\
\text { Hepáticas y Digestivas, Instituto de Salud Carlos III, }\end{array}$ \\
\hline Key Words: & $\begin{array}{l}\text { Ankyrin-like with transmembrane domains protein } 1 \text {, oropharyngeal } \\
\text { dysphagia, sensory input, vanilloid receptor } 1\end{array}$ \\
\hline
\end{tabular}




\section{Localization and expression of TRPV1 and TRPA1 in the human oropharynx and larynx}

Running head: TRPV1 and TRPA1 in the human oropharynx

D. Alvarez-Berdugo ${ }^{1}$, L. Rofes ${ }^{2}$, R. Farré ${ }^{2,3}$, JF. Casamitjana ${ }^{4}$, A. Enrique $^{4}$, J. Chamizo $^{4}$, A. Padrón 5 , X. Navarro ${ }^{6}$, P. Clavé $e^{1,2,7}$

1. Gastrointestinal Motility Laboratory, Hospital de Mataró, Consorci Sanitari del Maresme, Mataró, Spain.

2. Centro de Investigación Biomédica en Red de Enfermedades Hepáticas y Digestivas, Instituto de Salud Carlos III, Barcelona, Spain.

3. Translational Research Center for Gastrointestinal Disorders, KU Leuven University of Leuven, Leuven, Belgium.

4. ENT Department, Hospital de Mataró, Consorci Sanitari del Maresme, Mataró, Spain.

5. Department of Pathology, Hospital de Mataró, Consorci Sanitari del Maresme, Mataró, Spain.

6. Department of Cell Biology, Physiology and Immunology, Institute of Neurosciences, Universitat Autònoma de Barcelona, and Centro de Investigación Biomédica en Red sobre Enfermedades Neurodegenerativas, Bellaterra, Spain

7. Fundació Institut de Investigació Germans Trias i Pujol, Badalona, Spain

Correspondence: Pere Clavé, $\mathrm{MD}, \mathrm{PhD}$.

Associate Professor of Surgery.

Gastrointestinal Physiology Lab.

Department of Surgery. Hospital de Mataró.

Universitat Autònoma de Barcelona.

Carretera de Cirera s/n 08304.

Mataró. Spain.

Tel. +349374177 00.Fax. +34 937417733

E-mail: pere.clave@ciberehd.org 
D. Alvarez-Berdugo et al.

\begin{abstract}
Background. Previous studies have found that TRPV1 and TRPA1 receptor agonists improve

swallow response in patients with oropharyngeal dysphagia, but little is known about the expression of these receptors in the human oropharynx. The aim of this study was to assess the expression and localization of TRPV1 and TRPA1 in human samples from the oropharynx of healthy patients, to provide the basis for new pharmacological treatments for oropharyngeal dysphagia (OD).
\end{abstract}

Methods. Samples from oropharyngeal regions innervated by cranial nerves V, IX and X (tongue, pharynx and epiglottis) were obtained during ENT surgery and processed either for mRNA (21 patients) or for immunohistochemical assays (7 patients). The expression analysis was performed with RT-qPCR using ACTBh as reference gene. H\&E staining was used to study the histology; the immunohistochemical assay used a) neuron-specific enolase to detect nerve fibers or b) fluorescent probes to locate TRPV1 and TRPA1.

Results. TRPV1 was expressed in the three studied regions, with higher levels in CN V region (tongue) than in $\mathrm{CN} \mathrm{X}$ region (epiglottis) $(\mathrm{p}<0.05)$, and was localized at epithelial cells and nociceptive fibers in all studied regions. TRPA1 was also expressed in all studied regions but was always localized below the basal lamina. No immunoreactivity for TRPA1 was found on epithelial cells.

Conclusions. TRPV1 and TRPA1 are widely expressed in the human oropharynx with two distinct patterns. Our study further confirms that TRPV1/A1 receptors are promising therapeutic targets to develop active treatments for OD patients.

Keywords: Ankyrin-like with transmembrane domains protein 1, oropharyngeal dysphagia, sensory input, vanilloid receptor 1 . 
D. Alvarez-Berdugo et al.

\section{Key messages:}

- Previous studies have provided supportive evidence to suggest that TRPV1 and TRPA1 agonists improve swallow response on neurogenic OD patients, but little is known about the expression and localization of these receptors in the oropharynx.

- This study explores the expression and localization of TRPV1 and TRPA1 in human oropharyngeal samples with RT-qPCR and fluorescent immunohistochemistry.

- TRPV1 and TPRA1 expression is found throughout the oropharynx and these receptors are immunolocalized with two specific patterns. TRPV1 is found on epithelial cells and submucosal sensory nerves while TRPA1 is only found below the basal lamina.

- Oropharyngeal TRPV1/A1 receptors are promising therapeutic targets to develop active treatments for OD patients. 
D. Alvarez-Berdugo et al.

\section{Introduction.}

Oropharyngeal dysphagia (OD) is a geriatric syndrome affecting $23 \%$ of independently-living and up to $51 \%$ of institutionalized older people $(1,2,3)$. OD causes two groups of clinically relevant complications: decreased efficacy of swallowing that can lead to malnutrition and dehydration, and decreased safety of deglutition that can involve bolus penetration into the laryngeal vestibule and, in the most severe cases, tracheobronchial aspiration, leading to aspiration pneumonia (4,5). OD is also a highly frequent post-stroke complaint with an incidence of over $40 \%(6,7)$ and is a common condition in patients with neurodegenerative diseases such as Parkinson's and Alzheimer's disease, with reported prevalence of $35 \%-82 \%$ and $19 \%-84 \%$ respectively $(8,9)$. There is no pharmacological treatment for OD yet and its management is mainly based on compensatory strategies such as food and liquid bolus adaptation, postures, and swallow maneuvers $(10,11)$.

Old and neurogenic patients with OD present decreased sensitivity in the oropharynx $(12,13)$. Afferent myelinated nerve fibers in the superior laryngeal nerve have been observed to decrease with age and may be the cause of age-related sensory dysfunction of the oropharynx $(14,15)$. In stroke patients, lack of sensitivity could be caused by interference in the connection between the sensory afferents and the cortex and the brainstem (16) as well as the disruption of afferent information processing at the cortex due to cortical lesion. These sensory deficits may lead to delayed swallowing response (4) and predispose to aspiration and aspiration pneumonia (17).

It has been hypothesized that increasing the sensory input in these patients could modulate the cortical swallowing motor pathway and thus improve the swallowing response $(18,19)$. This hypothesis led us to perform clinical assays to test capsaicinoids (TRPV1 agonist) and piperine (TRPV1/A1 agonist) as a pharmacological approach to treat OD, with the results that swallow safety and efficacy were significantly increased by improved oropharyngeal motor 
D. Alvarez-Berdugo et al.

response $(20,21)$. However, more knowledge is needed about the mechanisms by which this approach works.

Caterina et al identified the transient receptor potential cation channel, subfamily $\mathrm{V}$, member 1 (TRPV1) as the vanilloid receptor (22) and, since then, TRPV1 expression has been found in several human tissues; in neuronal tissues such as the dorsal root ganglia (23) and the trigeminal ganglia (24), and also in epithelial cells like bladder epithelial cells in the human urothelium (25) and keratinocytes of human epidermis (26). Following TRPV1 identification, several polymodal receptors have been identified. Among them, Story et al identified the transient receptor potential cation channel, subfamily A, member 1 (TRPA1) as a cold activated receptor (27). TRPA1 is also activated by piperine and low concentrations of menthol $(28,29)$. However, little is known about the expression and localization of TRPV1 and TRPA1 within the human oropharynx.

The aim of this study was to explore the expression and localization of TRPV1 and TRPA1 in areas of the human oropharynx, including those innervated by trigeminal (cranial nerve V), glossopharyngeal (cranial nerve IX) and vagus (cranial nerve X) nerves. This knowledge may provide the basis to develop specific pharmacologic strategies with TRPV1 and TRPA1 agonists to treat patients with OD.

\section{Patients, materials and methods}

Sample collection. Human biopsies were obtained during routine major ENT surgical interventions once patients had granted informed consent. The samples were innervated by cranial nerves V (tongue), IX (faucial pillars and pharynx) and X (pharynx and epiglottis) with an average size of $8 \mathrm{~mm} \times 8 \mathrm{~mm} \times 4 \mathrm{~mm}$ for histological procedures and $3 \mathrm{~mm} \times 3 \mathrm{~mm} \times$ $3 \mathrm{~mm}$ for molecular biology procedures. The patients had head and neck cancer located at oral cavity $(21.4 \%)$, pharynx $(17.9 \%)$ or larynx $(53.6 \%)$, more than half of them at early stage 
D. Alvarez-Berdugo et al.

(53.8\% T1N0M0), or had undergone tonsillectomy for benign disease $(7.1 \%)$ and had not received chemo- or radiotherapy previous to the surgical intervention; all tissues used in the study were examined to confirm they were free from pathology. Samples from 7 patients (mean age $52.6 \pm 16.0,85.7 \%$ males) were processed to study both the histology and the innervation of the oropharynx and to localize TRPV1 and TRPA1 within tissues by immunohistochemistry. Samples from 21 patients (mean age $63.7 \pm 11.4,85.7 \%$ males) were processed to quantify TRPV1 and TRPA1 expression through RT-qPCR,

Tissue collection and storage were performed according to the recommendations from the "Human Tissue Act" of 21 October 2002 in the HHW Department of USA and obeying the current Spanish law about the use and storage of human tissue (Ley 14/2007 Título V Capítulo III). The Ethical Committee of the Consorci Sanitari del Maresme approved the study under the code $22 / 09$.

Histological sample preparation. Samples destined to immunohistochemistry were collected in paraformaldehyde $5 \%$ and incubated at $4^{\circ} \mathrm{C}$ for 1 to 2 hours. Biopsies were then cut in half, one part for colorimetric and the other for fluorescent immunohistochemistry procedures. The colorimetric immunohistochemistry samples were processed with the routine protocol of infiltration using the Tissue-Tek VIP Vacuum Infiltration Processor and reactives (Sakura Finetek, Tokyo, Japan) and then oriented by a pathologist and embedded in paraffin blocks. The samples were cut in $4 \mu \mathrm{m}$ slices at room temperature and processed. For fluorescent immunohistochemistry, samples were washed with phosphate buffer saline and incubated in cryoprotective solution $(20 \%$ sucrose in phosphate buffer $0.1 \mathrm{M})$ at $4^{\circ} \mathrm{C} \mathrm{O} / \mathrm{N}$, then frozen in Optimal Cutting Temperature compound (Sakura Finetek, Tokyo, Japan) and stored at $-80^{\circ} \mathrm{C}$ until used. The samples were cut in $6-10 \mu \mathrm{m}$ slices at $-20^{\circ} \mathrm{C}$ in a cryostat, set in FLEX IHC microscope slides (Dako, Glostrup, Denmark) and stored at $-20^{\circ} \mathrm{C}$ until used (30). 
D. Alvarez-Berdugo et al.

Colorimetric immunohistochemistry. Histological preparations were dyed with 1) hematoxylin eosin to examine the structure of oropharyngeal mucosa and submucosa using the Leica ST4020 device and reactives (Leica Biosystems, Nussloch, Germany) or 2) with colorimetric immunohistochemistry with the anti-Neuron-Specific Enolase antibody to find the location of nerve fibers using the AutostainerLink 48 (Dako, Glostrup, Denmark). Histological preparations were observed under a Nikon Eclipse E600 optical microscope (Nikon, Tokyo, Japan) and microscopic photographs were taken with an Olympus DP20 digital camera (Olympus, Tokyo, Japan).

Fluorescent immunohistochemistry. To locate TRP receptors, histological preparations were fixed with paraformaldehyde $4 \%$ in Hank's Balanced Salt Solution (HBSS) 0.1M, permeated with Triton $0.5 \%$, blocked with HBSS-goat serum 10\%, incubated with 1:500 dilution of anti-TRPV1 or anti-TRPA1 goat antibody (Alomone Labs, Jerusalem, Israel) diluted in HBSS-goat serum $10 \%$ at $4^{\circ} \mathrm{C} \mathrm{O} / \mathrm{N}$. They were washed and then incubated with $\mathrm{Rb}$ A568 (Thermo Fisher Scientific Inc., Carlsbad, USA) as secondary antibody diluted in HBSSGoat serum $10 \%$ at $37^{\circ} \mathrm{C}$ for 1 hour. Finally, preparations were incubated with Hoechst (Thermo Fisher Scientific Inc., Carlsbad, USA) and mounted with Fluoprep (BioMérieux, Rhône-Alpes, France). Histological preparations were observed and recorded with a Leica's laser confocal microscope SP5 (Leica Microsystems, Wetzlar, Germany) with 40x or 20x objectives. Three controls were performed for each antibody in each studied region: one using the antigenic peptide provided by the manufacturer at saturating concentration as blocking control, another without the primary antibody to check for unspecific binding of the secondary antibody and the last one without antibodies to check for tissue autofluorescence.

mRNA extraction. Samples intended for molecular biology were collected in RNAlater ${ }^{\circledR}$ solution (Thermo Fisher Scientific Inc., Carlsbad, USA), incubated O/N at $4^{\circ} \mathrm{C}$ and stored at - 
D. Alvarez-Berdugo et al.

$20^{\circ} \mathrm{C}$ until used. mRNA was extracted using TRIzol® (Thermo Fisher Scientific Inc., Carlsbad, USA) protocol, its purity and concentration was assessed with spectrophotometer and it was stored in DEPC-treated water (Thermo Fisher Scientific Inc., Carlsbad, USA) at $20^{\circ} \mathrm{C}$ until used.

Retrotranscription and RT-qPCR. Samples were treated with DNase I recombinant, RNase-free (Roche, Basel, Switzerland) to eliminate all traces of genomic DNA. mRNA was retrotranscribed using Transcriptor First Strand cDNA Synthesis Kit (Roche, Basel, Switzerland) to obtain cDNA. RT-qPCR was performed with a Roche's LightCycler ${ }^{\circledR} 2.0$ using LighCycler ${ }^{\circledR}$ TaqMan ${ }^{\circledR}$ Master kit (Roche, Basel, Switzerland). The PCR programs consisted of a 10 -minute, $95^{\circ} \mathrm{C}$ pre-incubation phase followed by 30 to 60 amplification cycles with an annealing temperature of $64^{\circ}$. The expression of human $\beta$-actin (ACTBh) as reference gene and TRPV1 and TRPA1 as target genes were analyzed by this method. All primers $(\underline{\text { Table } 1})$ were designed through the Universal Probe Library software (Roche, Basel, Switzerland).

Data analysis. TRPV1 and TRPA1 patterns of localization were analyzed using the mean intensity of signal of two consecutive $10 \times 10 \mu \mathrm{m}$ ROIs containing 700 pixels each. These mean intensities were graphed relative to the distance between the most superficial side of the ROIs and the basal lamina. The standard error was calculated for each of these means. TRPV1 and TRPA1 relative expressions to ACTBh were obtained using standard curves of the target and reference genes. The relative expression of all regions was compared using Kruskal-Wallis test and then Mann-Whitney U test was used to confirm differences between pairs; $p<0.05$ was considered as statistically significant. Results are expressed as the mean relative expression \pm standard error of the mean. Statistical analysis was performed with GraphPad Prism 5.0 (GraphPad Software Inc, San Diego, USA). 
D. Alvarez-Berdugo et al.

\section{Results}

\section{Colorimetric histology}

\section{1) Histology and innervation of the oropharynx}

Tongue mucosa had the characteristics of masticatory mucosa, with para-keratinized epithelium covering the filiform papillae (Fig 1A). Pharynx (Fig 1C) and epiglottis (Fig 1E) mucosa had the aspect of lining mucosa, with non-keratinized epithelia at the outer layers of mucosa. The basal lamina was identified between the epithelium and the submucosa in all regions. All regions showed abundant innervation consisting in thick fiber bundles at deep areas of the submucosa and single fibers approaching the epithelium, some of them reaching the basal lamina in tongue (Fig 1B) and lingual surface of epiglottis (Fig 1F). However, no nerve fibers were seen above the basal lamina in any sample of the studied regions.

\section{Fluorescent immunohistochemistry}

\section{2) TRPV1 localization.}

TRPV1 immunofluorescence was localized on the plasma membrane of the epithelial cells in all studied areas, showing a stronger signal in epithelial cells of the basal and intermediate layers and weaker signal on epithelial cells of more superficial and mature layers of the epithelium (Fig 2). TRPV1 was also found in nociceptive A $\delta$ fibers and polymorphonuclear leukocytes in the submucosa near the basal lamina (Fig 2C). The primary antibody control with specific blocking peptide showed no signal, suggesting that the immunoreactivity found is specific for TRPV1 (data not shown).

\section{3) TRPA1 localization}

TRPA1 was found below the basal lamina in all regions, on cells that morphologically resembled submucosal fibroblasts. No signal was found on epithelial cells (Fig 3). Specificity control of the primary antibody with blocking peptide showed no signal, suggesting that the immunoreactivity was specific for TRPA1 (data not shown). 
D. Alvarez-Berdugo et al.

\section{4) TRPV1/A1 localization patterns}

TRPV1 and TRPA1 showed two distinct patterns of localization in the three areas (CN V, IX, $\mathrm{X})$ of the human oropharynx: TRPV1 was localized in the epithelium with greater intensity near the basal lamina and gradually decreasing towards the superficial layers of the epithelium, and in nerve fibers of the submucosa. In contrast, TRPA1 was only localized in sparse cells and submucosal structures below the basal lamina; the morphology of immunoreactive cells resembled that of fibroblasts (Fig 4).

\section{RT-qPCR}

\section{5) Quantitative mRNA TRPV1 analysis.}

The relative expression of TRPV1 to $\beta$-actin was $25.43 \cdot 10^{-5} \pm 2.117 \cdot 10^{-5}$ in the region innervated by $\mathrm{CN} \mathrm{V}$ (tongue), $20.97 \cdot 10^{-5} \pm 2.056 \cdot 10^{-5}$ in the region innervated by $\mathrm{CN}$ IX and X (faucial pillars and pharynx) and $16.59 \cdot 10^{-5} \pm 1.494 \cdot 10^{-5}$ in the region innervated by CN X (lingual surface of epiglottis), showing a significantly higher relative expression of TRPV1 to $\beta$-actin in tongue than in epiglottis $(\mathrm{p}<0.05)($ Fig $5 \mathrm{~A})$.

\section{6) Quantitative mRNA TRPA1 analysis}

The relative expression of TRPA1 to $\beta$-actin was $2.736 \cdot 10^{-5} \pm 7.805 \cdot 10^{-6}$ in the region innervated by $\mathrm{CN} \mathrm{V}$ (tongue), $1.493 \cdot 10^{-5} \pm 2.684 \cdot 10^{-6}$ in the region innervated by $\mathrm{CN}$ IX (faucial pillars) and $2.432 \cdot 10^{-5} \pm 7.970 \cdot 10^{-6}$ in the region innervated by $\mathrm{CN} X$ (lingual surface of epiglottis), without significant differences between these areas (Fig 5A).

TRPA1 relative expression was significantly lower than TRPV1 relative expression in all the studied areas $(\mathrm{p}<0.001)$ (Fig 5A). The relative expression of TRPV1 to TRPA1 was $38.44 \pm 13.11$ in the region innervated by $\mathrm{CN} V$ (tongue), $16.71 \pm 3.24$ in the region innervated by CN IX (faucial pillars) and 27.14 \pm 9.52 in the region innervated by $\mathrm{CN} X$ (lingual surface of epiglottis), showing higher expression of TRPV1 in a consistent proportion in all areas (Fig 5B). Nevertheless, it should be noted that the biopsies contained proportionally more 
D. Alvarez-Berdugo et al.

epithelial cells expressing TRPV1 than subepithelial elements expressing TRPA1, which could affect the comparative assessment.

\section{Discussion}

Our results show that TRPV1 and TRPA1 channels are expressed throughout the regions innervated by the cranial nerves $\mathrm{V}$, IX and $\mathrm{X}$ and are localized in two distinct patterns: TRPV1 is localized in epithelial cells and a few sensory fibers in the oropharyngeal submucosa while TRPA1 is localized below the basal lamina but not in epithelial cells from the oropharynx.

The cranial nerves $\mathrm{V}$, IX and $\mathrm{X}$ provide oropharyngeal chemosensation through free endings of their axons. Signals elicited by stimuli travel along these axons to the cell bodies located in the corresponding ganglia and then continue to the spinal nucleus of the trigeminal complex of the brainstem (31).

Our results show how the oropharyngeal nerve fibers travel in bundles in deep layers of the submucosa while single axons reach the basal lamina and even contact the epithelial cells of the basal layer of the epithelium. However, no fibers were observed in the epithelium beyond the basal lamina. The presence of free nerve endings in the epithelium has been debated in the literature. Some studies have shown the presence of free nerve endings in contact with the basal lamina (32) and even penetrating it to reach superficial layers of the epithelium (33). The nerve endings found in our study might be the ones responding to chemical, thermal and noxious stimuli in the oropharynx.

Our results show the presence of TRPV1 on the epithelial cells of tongue, pharynx and epiglottis as well as in A $\delta$ fibers of the submucosa and TRPV1 expression in the regions 
D. Alvarez-Berdugo et al.

innervated by the cranial nerves V, IX and X. Furthermore, the results show decreasing expression of TRPV1 the lower the studied region, with significantly different levels of expression between tongue and epiglottis.

TRPV1 expression has been thoroughly examined in murine models, providing evidence of its presence both at the epithelial cells and in afferent fibers throughout the oropharynx $(34,35,36)$. However, there is less information on TRPV1 expression and localization in the human oropharynx. Hou et al found TRPV1 expression and immunoreactivity in human trigeminal ganglia neuronal cells and co-localization with CGRP and SP (37). TRPV1 has been also localized in peripheral free nerve endings of the human larynx (38) and tongue (39). Some studies have also found the expression and localization of TRPV1 in epithelial cells from the tongue $(39,40)$ where, as previously hypothesized, it could trigger the release of inflammatory mediators that would interact with neuronal terminals and so amplify the signaling (41).

From a functional point of view, it can be hypothesized that TRPV1 agonists, such as capsaicin, elicit their effect at both epithelial cells of the basal layer and afferent fibers in the submucosa. Capsaicin is a lipophilic molecule which permeates epithelial layers $(42,43)$ and so can travel through the epithelium and bind TRPV1 expressed by epithelial cells. This then activates $\mathrm{Ca}^{+2}$ inward currents in those cells which in turn activate the biosynthesis of endocannabinoids or other molecules known to modulate TRPV1 $(44,45)$ and are synthesized in the epithelium (46); these endogenous agonists, along with the exogenous TRPV1 agonists, activate TRPV1 on the nerve fibers. Localized effect of capsaicin is thus amplified by the epithelium to elicit its pungent sensation through the afferent fibers of the submucosa.

Regarding TRPA1, our results show TRPA1 immunoreactivity on the surface of cells, presumably fibroblasts, below the basal lamina in all studied regions but not in epithelial 
D. Alvarez-Berdugo et al.

cells. We found that TRPA1 expression is similar in all the regions innervated by the cranial nerves V, IX and X, and at considerably lower levels than the relative expression of TRPV1. Bautista et al described that the population of trigeminal ganglia neurons expressing TRPA1 represents $20 \%$ of small diameter axon neurons, all of which co-localized TRPA1 and TRPV1 while most of them also co-localized CGRP. They suggested that TRPA1 activates a subpopulation of peptidergic sensory neurons which are sensitive to capsaicin and produce peptidic inflammatory mediators (47). As for peripheral localization of TRPA1, previous studies have also found evidence of the presence of TRPA1 on nerves in human tongue (48) and pharynx (49) as well as in epithelial cells and fibroblasts of human skin and bronchial epithelia $(50,51)$. At these locations, it could be involved in inflammatory processes by stimulating the synthesis and release of cytokines such as IL-8 $(50,51,52)$.

As stated above, the lower TRPA1 expression relative to TRPV1 in the studied regions could be due to the fact that biopsies contain proportionally more epithelial cells expressing TRPV1 than subepithelial elements expressing TRPA1; further research should be done to determine the difference of expression between TRPV1 and TRPA1 in peripheral tissues of the oropharynx.

Several studies have pointed out that oropharyngeal sensitivity is reduced in older people due to a decrease in myelinated sensory fibers $(12,14,15)$. This study has not checked the effect of age on the expression of TRPV1 and TRPA1, but considers it would be of great interest to assess the effect of age on expression in future research.

In summary, the present study confirms the presence of TRPV1 and TRPA1 in the regions where capsaicinoids and piperine were thought to elicit their pharmacological effect by enhancing afferent sensory inputs to the cortex and swallowing centers (20,21). The study presents two distinct patterns for the localization of TRPV1 and TRPA1: TRPV1 is found within epithelial cells of the oropharynx while TRPA1 is found below the basal lamina. The 
D. Alvarez-Berdugo et al.

anatomical and histological map of TRPV1 and TRPA1 expression may help to explain the way TRPV1 and TRPA1 respond to stimuli from both capsaicinoids and piperine and set the basis for future use of both agonists to treat OD. These studies should also consider the possible chemical desensitization of these receptors after continuous long term exposure to $\underline{\text { high doses of the agonists. }}$

\section{Acknowledgements}

This study was supported by grants from the Spanish Ministerio de Ciencia e Innovación (PS09/01012, INT 10/228), the Agencia de Gestió d'Ajuts Universitaris i de Recerca (2009 SGR 708), and by the Fundació la Marató de TV3 (Project 112310). The authors thank the head of the Department of Pathology, Carme Admella, and the technicians Silvia García, Estibaliz Hinchada and Mari Pau Pérez for their assessment and assistance in histological procedures. They would also like to thank Mónica Roldán and Meritxell Vendrell for their assistance with the fluorescent IHC and the laser confocal microscope, Irene López for her assistance with the molecular biology procedures, and Marta Morell for assistance in sample processing. The authors thank Jane Lewis for writing assistance. 
D. Alvarez-Berdugo et al.

\section{Reference List}

1. Serra-Prat M, Hinojosa G, Lopez D et al. Prevalence of Oropharyngeal Dysphagia and Impaired Safety and Efficacy of Swallow in Independently Living Older Persons. Journal of the American Geriatrics Society 2011; 59: 186-187.

2. Lin LC, Wu SC, Chen HS, Wang TG, Chen MY. Prevalence of impaired swallowing in institutionalized older people in Taiwan. Journal of the American Geriatrics Society 2002; 50: 1118-1123.

3. Clave P, Shaker R. Dysphagia: current reality and scope of the problem. Nature Reviews Gastroenterology and Hepatology 2015; in press (doi: 10.1038/nrgastro.2015.49).

4. Rofes L, Arreola V, Romea M et al. Pathophysiology of oropharyngeal dysphagia in the frail elderly. Neurogastroenterology and Motility 2010; 22: 851-E230.

5. Serra-Prat M, Palomera M, Gomez C et al. Oropharyngeal dysphagia as a risk factor for malnutrition and lower respiratory tract infection in independently living older persons: a population-based prospective study. Age and Ageing 2012; 41: 376-381.

6. Rofes L, Vilardell N, Clave P. Post-stroke dysphagia: progress at last. Neurogastroenterology and Motility 2013; 25: 278-282.

7. Martino R, Foley N, Bhogal S, Diamant N, Speechley M, Teasell R. Dysphagia after stroke - Incidence, diagnosis, and pulmonary complications. Stroke 2005; 36: 27562763.

8. Kalf JG, de Swart BJM, Bloem BR, Munneke M. Prevalence of oropharyngeal dysphagia in Parkinson's disease: A meta-analysis. Parkinsonism \& Related Disorders 2012; 18: 311-315.

9. Langmore SE, Olney RK, Lomen-Hoerth C, Miller BL. Dysphagia in patients with frontotemporal lobar dementia. Archives of Neurology 2007; 64: 58-62.

10. Clave P, De Kraa M, Arreola V et al. The effect of bolus viscosity on swallowing function in neurogenic dysphagia. Alimentary Pharmacology \& Therapeutics 2006; 24: $1385-1394$.

11. Speyer R, Baijens L, Heijnen M, Zwijnenberg I. Effects of Therapy in Oropharyngeal Dysphagia by Speech and Language Therapists: A Systematic Review. Dysphagia 2010; 25: 40-65.

12. Aviv JE. Effects of aging on sensitivity of the pharyngeal and supraglottic areas. American Journal of Medicine 1997; 103: 74S-76S.

13. Aviv JE, Martin JH, Sacco RL et al. Supraglottic and pharyngeal sensory abnormalities in stroke patients with dysphagia. Annals of Otology Rhinology and Laryngology 1996; 105: 92-97.

14. Mortelliti AJ, Malmgren LT, Gacek RR. Ultrastructural-Changes with Age in the Human Superior Laryngeal Nerve. Archives of Otolaryngology-Head \& Neck Surgery 1990; 116: 1062-1069. 
D. Alvarez-Berdugo et al.

15. Tiago R, Pontes P, do Brasil OC. Age-related changes in human laryngeal nerves. Otolaryngology-Head and Neck Surgery 2007; 136: 747-751.

16. Teismann IK, Steinstraeter O, Stoeckigt $\mathrm{K}$ et al. Functional oropharyngeal sensory disruption interferes with the cortical control of swallowing. Bmc Neuroscience 2007; 8:

17. Almirall J, Rofes L, Serra-Prat $M$ et al. Oropharyngeal dysphagia is a risk factor for community-acquired pneumonia in the elderly. European Respiratory Journal 2013; 41: 923-928.

18. Teismann IK, Steinstrater $\mathrm{O}$, Warnecke $\mathrm{T}$ et al. Tactile thermal oral stimulation increases the cortical representation of swallowing. Bmc Neuroscience 2009; 10:

19. Hamdy S, Aziz Q, Rothwell JC, Hobson A, Barlow J, Thompson DG. Cranial nerve modulation of human cortical swallowing motor pathways. American Journal of Physiology-Gastrointestinal and Liver Physiology 1997; 272: G802-G808.

20. Rofes L, Arreola V, Martin A, Clave P. Natural capsaicinoids improve swallow response in older patients with oropharyngeal dysphagia. Gut 2013; 62: 1280-1287.

21. Rofes L, Arreola V, Martin A, Clave P. Effect of oral piperine on the swallow response of patients with oropharyngeal dysphagia. Journal of Gastroenterology 2014; 49: 15171523.

22. Caterina MJ, Schumacher MA, Tominaga M, Rosen TA, Levine JD, Julius D. The capsaicin receptor: a heat-activated ion channel in the pain pathway. Nature $1997 ; \mathbf{3 8 9}$ : 816-824.

23. Cortright DN, Crandall M, Sanchez JF, Zou T, Krause JE, White G. The tissue distribution and functional characterization of human VR1. Biochemical and Biophysical Research Communications 2001; 281: 1183-1189.

24. Ugawa S, Ueda T, Yamamura H, Nagao M, Shimada S. Coexpression of vanilloid receptor subtype-1 and acid-sensing ion channel genes in the human trigeminal ganglion neurons. Chemical Senses 2005; 30: I195-I195.

25. Shabir S, Cross W, Kirkwood LA et al. Functional expression of purinergic P2 receptors and transient receptor potential channels by the human urothelium. American Journal of Physiology-Renal Physiology 2013; 305: F396-F406.

26. Denda $\mathrm{M}$, Fuziwara $\mathrm{S}$, Inoue $\mathrm{K}$ et al. Immunoreactivity of VR1 on epidermal keratinocyte of human skin. Biochemical and Biophysical Research Communications 2001; 285: 1250-1252.

27. Story GM, Peier AM, Reeve AJ et al. ANKTM1, a TRP-like channel expressed in nociceptive neurons, is activated by cold temperatures. Cell 2003; 112: 819-829.

28. Okumura Y, Narukawa M, Iwasaki Y et al. Activation of TRPV1 and TRPA1 by Black Pepper Components. Bioscience Biotechnology and Biochemistry 2010; 74: 1068-1072.

29. Karashima Y, Damann N, Prenen J et al. Bimodal action of menthol on the transient receptor potential channel TRPA1. Journal of Neuroscience 2007; 27: 9874-9884. 
D. Alvarez-Berdugo et al.

30. Mager SR, Oomen MHA, Morente MM et al. Standard operating procedure for the collection of fresh frozen tissue samples. European Journal of Cancer 2007; 43: 828834.

31. Purve D, Augustine GJ, Fitzpatrick D, Hall WC, LaMantia AS, White LE, eds. Neuroscience, 5th edn. Sinauer Associates Inc, 2012

32. Bengoechea Gonzalez ME, Alvarez Arenal A, Perez Casas A, Suarez Garnacho S, Vega Alvarez A, Villa Vigil A. Microscopic innervation and nerve receptors of the lingual mucosa. Rev Eur Odontoestomatol 1989; 1: 123-130.

33. Munger BL. Intraepidermal Innervation of Snout Skin of Opossum - A Light and Electron Microscope Study with Observations on Nature of Merkels Tastzellen. Journal of Cell Biology 1965; 26: 79-\&.

34. Kido MA, Muroya H, Yamaza T, Terada Y, Tanaka T. Vanilloid receptor expression in the rat tongue and palate. Journal of Dental Research 2003; 82: 393-397.

35. Sasaki R, Sato T, Yajima T, Kano M, Suzuki T, Ichikawa H. The Distribution of TRPV1 and TRPV2 in the Rat Pharynx. Cellular and Molecular Neurobiology 2013; 33: $707-714$.

36. Kawashima M, Imura K, Sato I. Topographical organization of TRPV1-immunoreactive epithelium and CGRP-immunoreactive nerve terminals in rodent tongue. European Journal of Histochemistry 2012; 56: 129-134.

37. Hou MY, Uddman R, Tajti J, Kanje M, Edvinsson L. Capsaicin receptor immunoreactivity in the human trigeminal ganglion. Neuroscience Letters 2002; 330: 223-226.

38. Hamamoto T, Takumida M, Hirakawa K, Tatsukawa T, Ishibashi T. Localization of transient receptor potential vanilloid (TRPV) in the human larynx. Acta OtoLaryngologica 2009; 129: 560-568.

39. Tachibana T, Chiba H. Relationship between glossodynia and capsaicin sensitive sensory neurons. Journal of the Japanese Stomatology Society 2006; 55: 167-178.

40. Marincsak R, Toth BI, Czifra G et al. Increased expression of TRPV1 in squamous cell carcinoma of the human tongue. Oral Diseases 2009; 15: 328-335.

41. Southall MD, Li T, Gharibova LS, Pei Y, Nicol GD, Travers JB. Activation of epidermal vanilloid receptor-1 induces release of proinflammatory mediators in human keratinocytes. Journal of Pharmacology and Experimental Therapeutics 2003; 304: 217-222.

42. Pershing LK, Reilly CA, Corlett JL, Crouch DJ. Effects of vehicle on the uptake and elimination kinetics of capsaicinoids in human skin in vivo. Toxicology and Applied Pharmacology 2004; 200: 73-81.

43. Magnusson BM, Koskinen LOD. In vitro percutaneous penetration of topically applied capsaicin in relation to in vivo sensation responses. International Journal of Pharmaceutics 2000; 195: 55-62. 
D. Alvarez-Berdugo et al.

44. Zygmunt PM, Petersson J, Andersson DA et al. Vanilloid receptors on sensory nerves mediate the vasodilator action of anandamide. Nature 1999; 400: 452-457.

45. Ahluwalia J, Urban L, Bevan S, Nagy I. Anandamide regulates neuropeptide release from capsaicin-sensitive primary sensory neurons by activating both the cannabinoid 1 receptor and the vanilloid receptor 1 in vitro. European Journal of Neuroscience 2003; 17: 2611-2618.

46. Maccarrone $\mathrm{M}$, Di Rienzo M, Battista $\mathrm{N}$ et al. The endocannabinoid system in human keratinocytes - Evidence that anandamide inhibits epidermal differentiation through CB1 receptordependent inhibition of protein kinase $\mathrm{C}$, activating protein-1, and transglutaminase. Journal of Biological Chemistry 2003; 278: 33896-33903.

47. Bautista DM, Movahed P, Hinman A et al. Pungent products from garlic activate the sensory ion channel TRPA1. Proceedings of the National Academy of Sciences of the United States of America 2005; 102: 12248-12252.

48. Morgan CR, Bird EV, Robinson PP, Boissonade FM. TRPA1 expression in human lingual nerve neuromas in patients with and without symptoms of dysaesthesia. Neuroscience Letters 2009; 465: 189-193.

49. des Gachons CP, Uchida K, Bryant B et al. Unusual Pungency from Extra-Virgin Olive Oil Is Attributable to Restricted Spatial Expression of the Receptor of Oleocanthal. Journal of Neuroscience 2011; 31: 999-1009.

50. Atoyan R, Shander D, Botchkareva NV. Non-Neuronal Expression of Transient Receptor Potential Type A1 (TRPA1) in Human Skin. Journal of Investigative Dermatology 2009; 129: 2312-2315.

51. Mukhopadhyay I, Gomes P, Aranake S et al. Expression of functional TRPA1 receptor on human lung fibroblast and epithelial cells. Journal of Receptors and Signal Transduction 2011; 31: 350-358.

52. Biro T, Kovacs L. An "Ice-Cold" TR(i)P to Skin Biology: The Role of TRPA1 in Human Epidermal Keratinocytes. Journal of Investigative Dermatology 2009; 129: 2096-2099. 
D. Alvarez-Berdugo et al.

\section{Tables}

\begin{tabular}{llll} 
Antibody & Deseription & Dilution & Secondary \\
\hline Anti-TRPV1 & Rabbit polyclonal (Alomone Labs, & $1: 500$ & Rb-A568 (Thermo Fisher \\
& Jerusalem, Israel) & & Scientific Inc., Carlsbad, USA) \\
Anti-TRPA1 & $\begin{array}{l}\text { Rabbit polyclonal (Alomone Labs, } \\
\text { Jerusalem, Israel) }\end{array}$ & & $\begin{array}{l}\text { Rb-A568 (Thermo Fisher } \\
\text { Scientific Inc., Carlsbad, USA) }\end{array}$
\end{tabular}

Table 1. Primary and secondary antibodies used for the fluorescent immunohistochemical assays.

\begin{tabular}{|c|c|c|}
\hline Gene & UPL & Primers \\
\hline$\beta$-actin (ACTBh) & 64 & $\begin{array}{l}\text { Left: 5' ccaaccgcgagaagatga 3' } \\
\text { Right: 5' tccatcacgatgccagtg 3' }\end{array}$ \\
\hline $\begin{array}{l}\text { TRP subfamily V member } 1 \\
\text { (TRPV1) }\end{array}$ & 41 & $\begin{array}{l}\text { Left: 5' cagctacagtgagatgctttctt 3', } \\
\text { Right: 5' atggaagccacatactcettg 3' }\end{array}$ \\
\hline $\begin{array}{l}\text { TRP subfamily A member } 1 \\
\text { (TRPA1) }\end{array}$ & 32 & $\begin{array}{l}\text { Left: } 5 \text { ' tcaccatgagctagcagactattt 3' } \\
\text { Right: } 5 \text { ' gagagcgtccttcagaatcg 3' }\end{array}$ \\
\hline
\end{tabular}

Table 1. Primers used for the RT-qPCR. TRP stands for transient receptor potential channel. UPL stands for Universal Probe Library. 
D. Alvarez-Berdugo et al.

\section{Figure legends}

Figure 1. Hematoxylin and eosin stain (HE) (top) and Neuronal Specific Enolase (NSE) (bottom) histological preparations under optical microscope. A Mucosa and submucosa of the mid third of the tongue with para-keratinized epithelia over the filiform papillae. B Region near A; most nerve fibers travel below the epithelium (arrowheads) and some nerve fibers contact the epithelial cells of the basal layer (arrow). C and E Lining mucosa of the posterior wall of the pharynx and the lingual surface of the epiglottis with non-keratinized epithelia. D Region near C; a single nerve fiber runs below the basal lamina (arrowhead). F Region near $\mathrm{E}$; single fibers and fiber bundles run below the basal lamina (arrowheads) and a sensory nerve structure contacts it (arrow). Top images were obtained under 100x and bars measure $50 \mu \mathrm{m}$; bottom images were obtained under $200 \mathrm{x}$ and bars measure $30 \mu \mathrm{m}$.

Figure 2. TRPV1 (red) immunoreactivity (IR) in fluorescent immunohistochemistry preparations with Hoechst staining of nuclei (blue) under confocal microscope. A Tongue mucosa and submucosa with TRPV1 IR on the membrane of epithelial cells from the basal layer of the epithelium; signal progressively fades in outer layers of the epithelium. B Pharynx mucosa and submucosa with intense TRPV1 IR on the membrane of epithelial cells from the basal layer of the epithelium; weaker TRPV1 IR is seen under the epithelium on blood vessel (asterisk) endothelial cells. C Epiglottis mucosa and submucosa with TRPV1 IR on the membrane of epithelial cells from the basal layer of the epithelium; TRPV1 IR can also

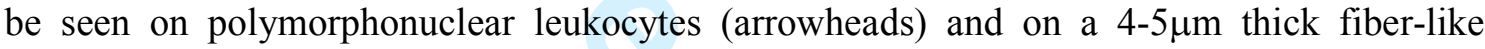
structure resembling an $A \delta$ nerve fiber (arrow). Images were obtained under 400x. Bars measure $30 \mu \mathrm{m}$.

Figure 3. TRPA1 (red) immunoreactivity (IR) in fluorescent immunohistochemistry preparations with Hoechst staining of nuclei (blue) under confocal microscope. A and B Tongue mucosa and submucosa with TRPA1 IR on subepithelial elements, both between the filiform papillae and deeper into the submucosa. C, D, E and F Pharynx (C and D) and epiglottis ( $\mathrm{E}$ and F) mucosa and submucosa with TRPA1 IR on scattered subepithelial elements. No signal is found on epithelial cells in any of the images. Images were obtained

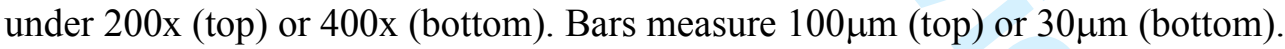

Figure 4. TRPV1 and TRPA1 distribution pattern. A Schematic drawing of the distribution pattern of TRPV1 (red) and TRPA1 (green) in the oropharynx. Nerves and fibroblasts are depicted in the submucosa. B Fluorescence intensity for TRPV1 (red) and TRPA1 (green) relative to the distance from the basal lamina of the epithelium of the oropharyngeal mucosa. Values of the $\mathrm{X}$ axis correspond to the distance between the most superficial side of the ROI and the basal lamina. Positive values of the $\mathrm{X}$ axis correspond to positions above the basal lamina (epithelium) and negative values correspond to positions below the basal lamina (submucosa). Intensity values are given as the mean of two correlative $10 \times 10 \mu \mathrm{m}$ regions of interest (ROIs). The SEM of each bar is displayed. A straight line is drawn in the position 0 of the $\mathrm{X}$ axis corresponding to the basal lamina.

Figure 5. TRPV1 and TRPA1 expression analysis. A Relative expression of TRPV1 (right) and TRPA1 (left) to $\beta$-actin (ACTBh) in the areas innervated by the trigeminal (CN V), glossopharyngeal (CN IX) and vagus $(\mathrm{CN} \mathrm{X})$ nerves. ${ }^{*} \mathrm{p}<0.05$. B Relative expression of TRPV1 to TRPA1 in the areas innervated by the trigeminal $(\mathrm{CN} \mathrm{V})$, glossopharyngeal $(\mathrm{CN}$ $\mathrm{IX})$ and vagus $(\mathrm{CN} \mathrm{X})$ nerves. 

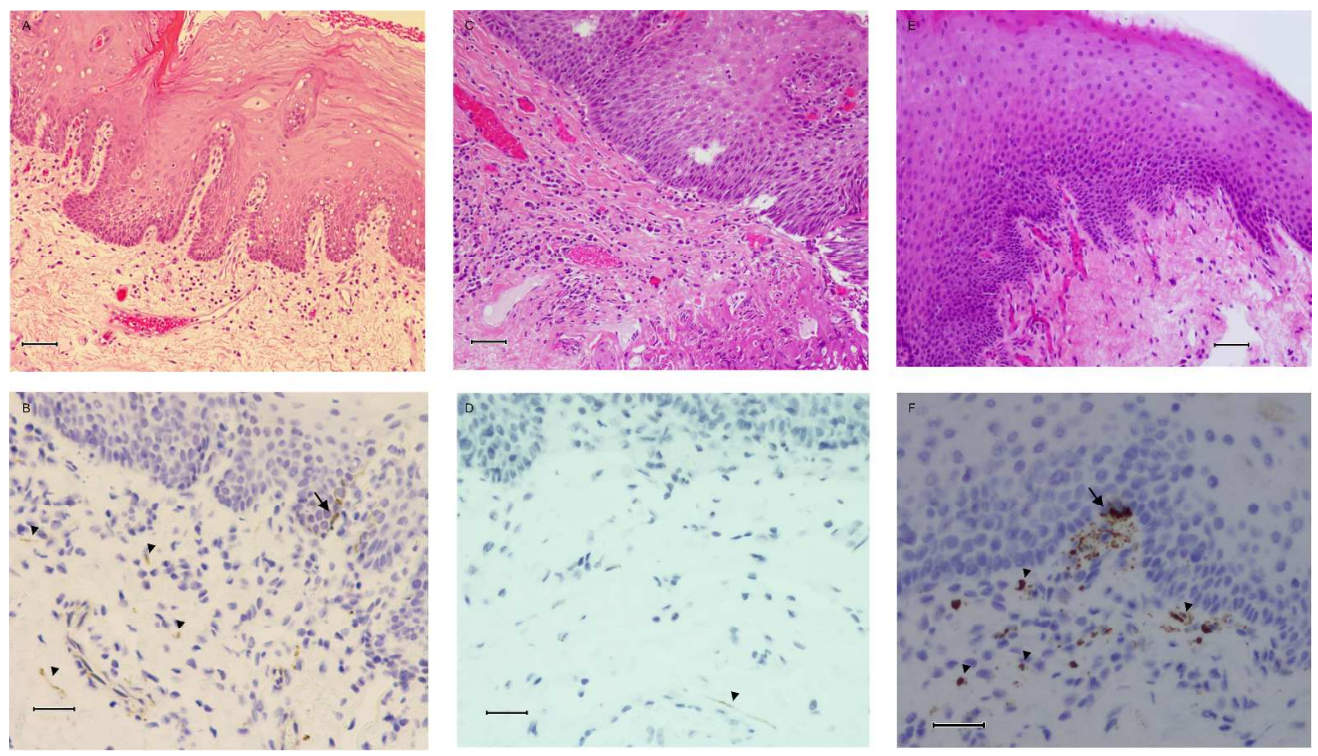

Hematoxylin and eosin stain (HE) (top) and Neuronal Specific Enolase (NSE) (bottom) histological preparations under optical microscope. A Mucosa and submucosa of the mid third of the tongue with parakeratinized epithelia over the filiform papillae. B Region near A; most nerve fibers travel below the epithelium (arrowheads) and some nerve fibers contact the epithelial cells of the basal layer (arrow). C and E Lining mucosa of the posterior wall of the pharynx and the lingual surface of the epiglottis with nonkeratinized epithelia. D Region near C; a single nerve fiber runs below the basal lamina (arrowhead). F Region near $\mathrm{E}$; single fibers and fiber bundles run below the basal lamina (arrowheads) and a sensory nerve structure contacts it (arrow). Top images were obtained under 100x and bars measure $50 \mu \mathrm{m}$; bottom images were obtained under $200 x$ and bars measure $30 \mu \mathrm{m}$. 

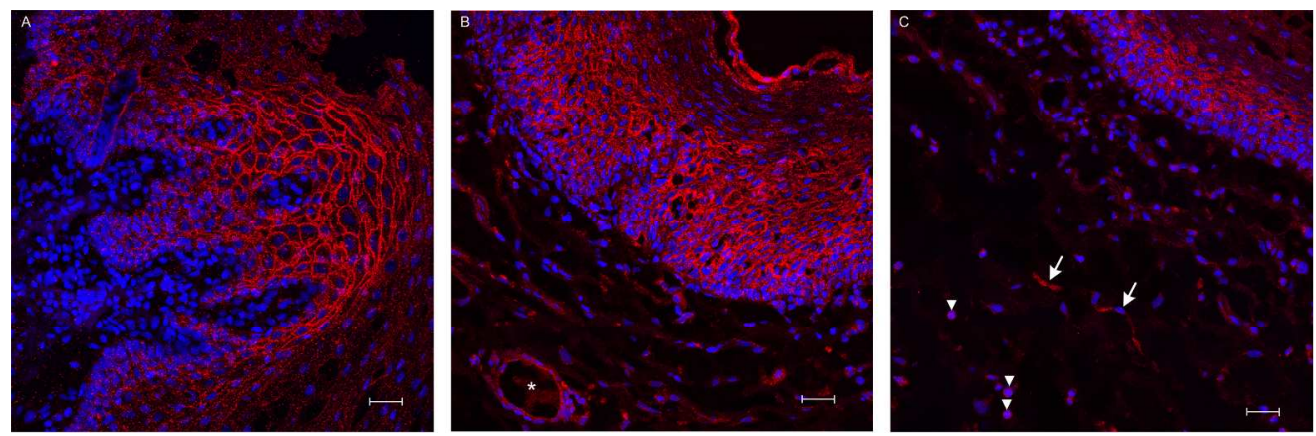

TRPV1 (red) immunoreactivity (IR) in fluorescent immunohistochemistry preparations with Hoechst staining of nuclei (blue) under confocal microscope. A Tongue mucosa and submucosa with TRPV1 IR on the membrane of epithelial cells from the basal layer of the epithelium; signal progressively fades in outer layers of the epithelium. B Pharynx mucosa and submucosa with intense TRPV1 IR on the membrane of epithelial cells from the basal layer of the epithelium; weaker TRPV1 IR is seen under the epithelium on blood vessel

(asterisk) endothelial cells. C Epiglottis mucosa and submucosa with TRPV1 IR on the membrane of epithelial cells from the basal layer of the epithelium; TRPV1 IR can also be seen on polymorphonuclear

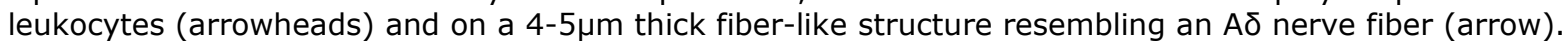

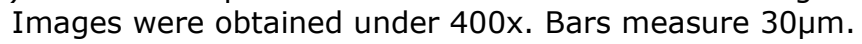



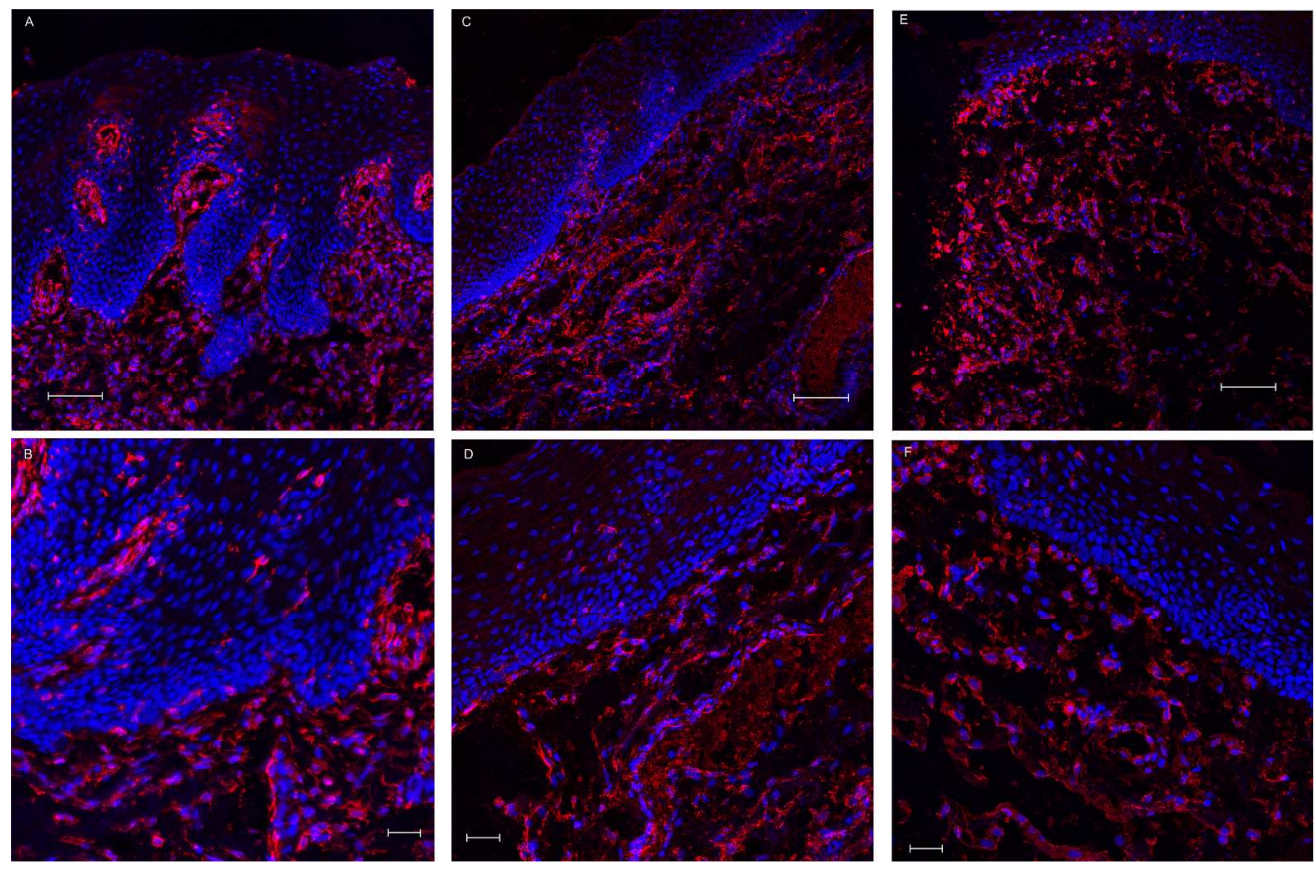

TRPA1 (red) immunoreactivity (IR) in fluorescent immunohistochemistry preparations with Hoechst staining of nuclei (blue) under confocal microscope. A and B Tongue mucosa and submucosa with TRPA1 IR on subepithelial elements, both between the filiform papillae and deeper into the submucosa. C, D, E and $\mathbf{F}$ Pharynx (C and D) and epiglottis (E and F) mucosa and submucosa with TRPA1 IR on scattered subepithelial elements. No signal is found on epithelial cells in any of the images. Images were obtained under 200x (top) or $400 x$ (bottom). Bars measure $100 \mu \mathrm{m}$ (top) or $30 \mu \mathrm{m}$ (bottom). $274 \times 180 \mathrm{~mm}(300 \times 300 \mathrm{DPI})$ 

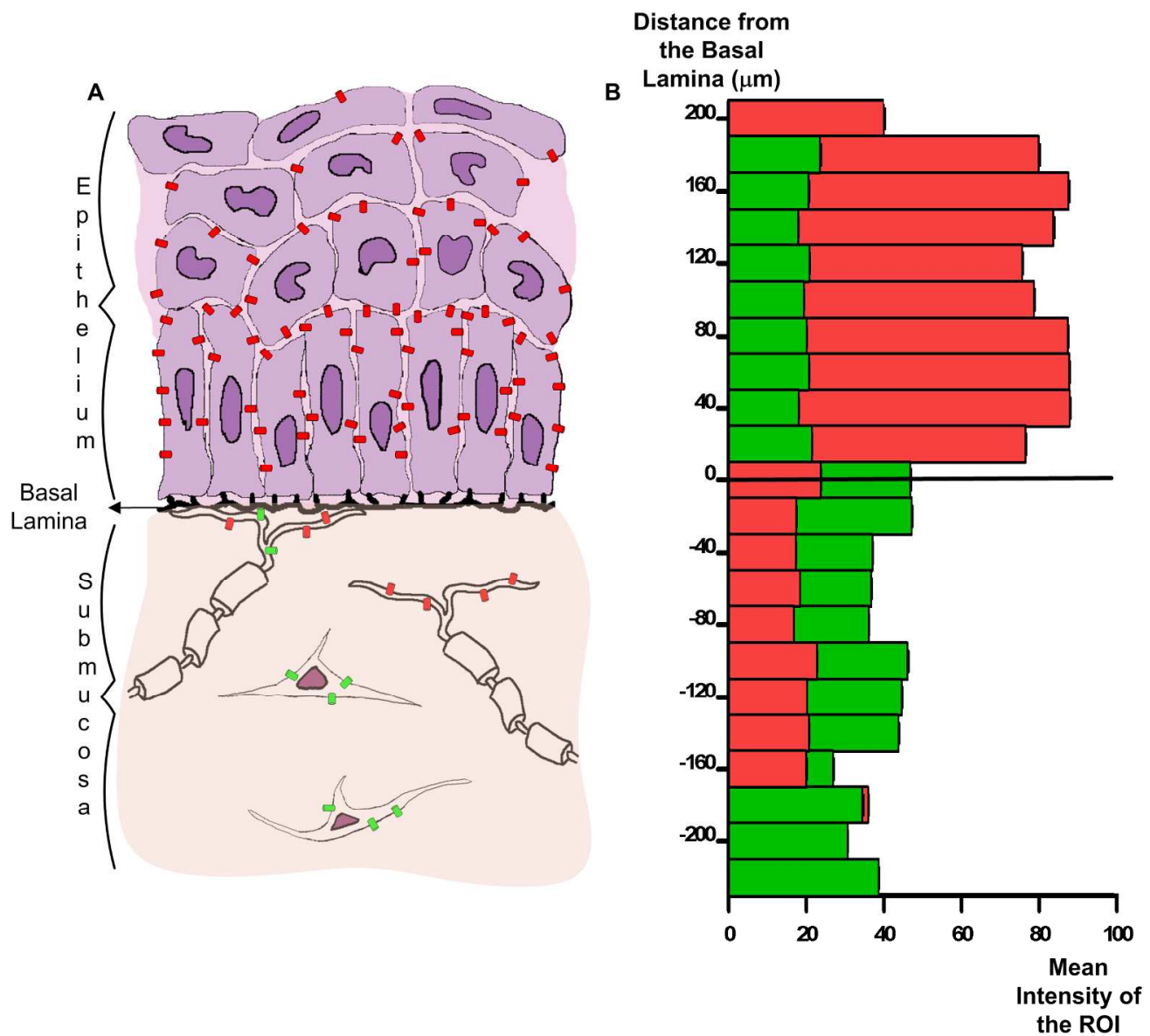

TRPV1 and TRPA1 distribution pattern. A Schematic drawing of the distribution pattern of TRPV1 (red) and TRPA1 (green) in the oropharynx. Nerves and fibroblasts are depicted in the submucosa. B Fluorescence intensity for TRPV1 (red) and TRPA1 (green) relative to the distance from the basal lamina of the epithelium of the oropharyngeal mucosa. Values of the $X$ axis correspond to the distance between the most superficial side of the ROI and the basal lamina. Positive values of the $X$ axis correspond to positions above the basal lamina (epithelium) and negative values correspond to positions below the basal lamina (submucosa). Intensity values are given as the mean of two correlative $10 \times 10 \mu \mathrm{m}$ regions of interest (ROIs). The SEM of each bar is displayed. A straight line is drawn in the position 0 of the $\mathrm{X}$ axis corresponding to the basal lamina. 

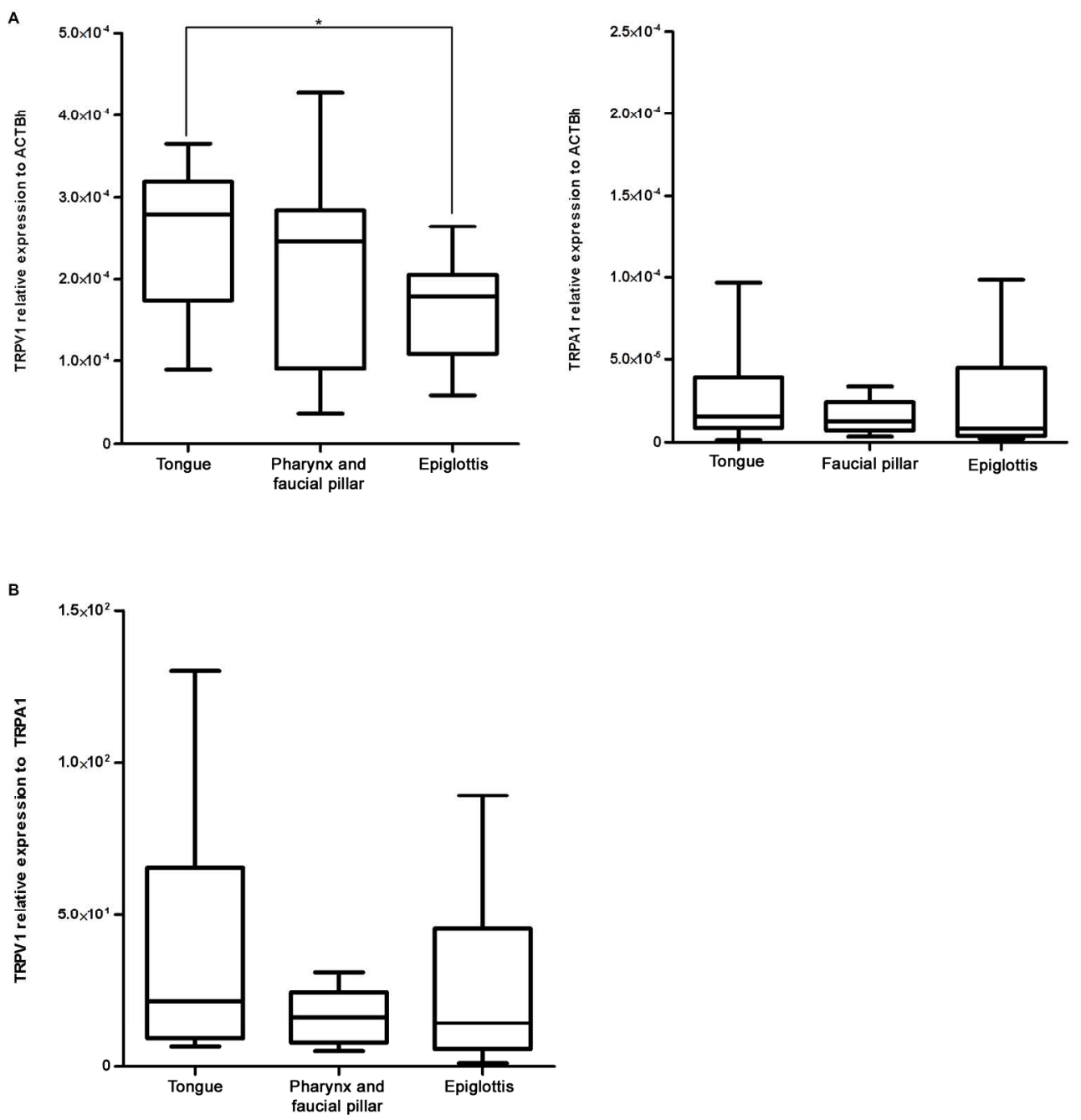

Figure 5. TRPV1 and TRPA1 expression analysis. A Relative expression of TRPV1 (right) and TRPA1 (left) to $\beta$-actin (ACTBh) in the areas innervated by the trigeminal (CN V), glossopharyngeal (CN IX) and vagus (CN $\mathrm{X}$ ) nerves. $* \mathrm{p}<0.05$. B Relative expression of TRPV1 to TRPA1 in the areas innervated by the trigeminal $(\mathrm{CN} \mathrm{V})$, glossopharyngeal (CN IX) and vagus (CN X) nerves. $190 \times 199 \mathrm{~mm}(300 \times 300 \mathrm{DPI})$ 